\title{
La televisión y pautas de conducta de los jóvenes salvadoreños
}

\author{
Edith Ruth Vaquerano de Portillo \\ edit_portillo@utec.edu.sv \\ Domingo Orlando Alfaro Alfaro \\ Escuela de Comunicaciones, Universidad Tecnológica de El Salvador
}

\section{Resumen}

Este artículo es producto de una investigación correlacional descriptiva realizada entre 1.207 jóvenes salvadoreños, con el objetivo de determinar si existe relación entre sus hábitos de consumo televisivo y sus pautas de conducta. Los resultados apuntan a la televisión como medio dominante - en el que se busca sobre todo entretenimiento-, con la Internet afianzándose en un sólido segundo lugar. Sin embargo, esa preferencia por el medio, aun y cuando los datos obtenidos permiten percibir una asociación entre su consumo y la manera en la que los jóvenes hablan, se comportan, lucen o piensan, no llegan a establecerse correlaciones estadísticas, con lo que habría que virar la vista hacia los hechos de que los receptores tienen un papel activo, de que la influencia de la televisión no es una variable unidimensional, y de que las interacciones sociales y el contexto deben ser considerados en profundidad. Para ello, es necesario recurrir a otros enfoques en la investigación, al estudio cualitativo. No obstante, la adopción de estrategias de educación para los medios, y especialmente para el consumo de la televisión, es una necesidad que se impone.

Palabras clave: Conducta del adolescente, televisión, investigación científica, estudio de los medios, educación para medios, mediación televisiva, pautas de conducta.

\begin{abstract}
This paper is the result of a descriptive correlational research, conducted among 1,207 young Salvadorans, in order to determine whether a relationship exists between television consumption habits and patterns of behavior. The results point to television as the dominant medium - seeking specially entertainment- followed by the Internet in a strong second place. However, this preference for television, even when the data allow us to perceive an association between consumption and the way young people speak, act, look or think, fail to establish statistical correlations, which should make us turn our eyes to the facts that the receivers have an active role, that the influence of television is not an unidimensional variable, and that social interactions and context must be considered in depth. For this it is necessary to resort to other approaches in research, to qualitative study. However, the adoption of strategies for media education, especially for television consumption, is an unavoidable necessity.
\end{abstract}

Keywords: Adolescent behavior, TV, scientific research, studies of the Media, education for the Media, televise, ion mediation, conduct guidelines. 


\section{Introducción}

Los medios de comunicación masiva, especialmente la televisión, son señalados frecuentemente como actores principales en la adquisición de pautas de conducta por parte de niños, adolescentes y jóvenes, ${ }^{1}$ al considerarlos, en tanto actores destacados de la llamada "escuela paralela,"2 como responsables de la transmisión tanto de valores como de contravalores.

Este impacto de los medios de comunicación en la formación de los jóvenes se explicaría por los volúmenes de información que comunican, superiores en cantidad a la que proviene del sistema de educación formal, lo que se vería favorecido además por la ausencia de sentido crítico en los jóvenes, así como por la falta de una educación en medios de comunicación. Con o sin intención, los medios de comunicación social se han vuelto el referente y vehículo por medio de los cuales las personas construyen su visión de la sociedad en que viven y las normas que la rigen, en una acción de socialización que no necesariamente coincide con las realizadas por la familia y la escuela.

No puede ignorarse el papel socializador de los medios de comunicación social, por lo que, conocer de qué manera y qué tanto influyen en la conducta de los jóvenes, reforzando o contradiciendo la labor realizada en la familia y en el aula, posibilitaría la toma de decisiones e impulso de acciones desde el hogar y la escuela, tendientes en esta última a incorporarlos sistemáticamente como recursos de aprendizaje.

En ese contexto, esta investigación se planteó como objetivo general determinar si existe relación entre los hábitos de consumo televisivo de los jóvenes salvadoreños y sus pautas de conducta. Como objetivos específicos se establecieron tres: el primero, identificar los hábitos de consumo televisivo de los jóvenes salvadoreños; el segundo, identificar la percepción que tienen los jóvenes salvadoreños sobre los contenidos de sus espacios televisivos preferidos; y el tercero, identificar la relación existente entre el consumo televisivo y las pautas de conducta de los jóvenes salvadoreños.

El estudio de los medios masivos de comunicación -y dentro de ellos, de la televisión- giró en la primera mitad del siglo $X X$ en torno a una visión psicosociológica conductista del efecto e influencia directos, que Laswell ${ }^{3}$ inmortalizó en la figura de la "aguja hipodérmica", en un modelo en el que los medios actuaban como inyecciones en las mentes de una audiencia pasiva y producían ciertos tipos de comportamientos.

En esa línea de pensamiento, la influencia de la televisión depende de dos aspectos: la exposición y el contenido, entre los cuales llega a establecer una relación directamente proporcional: cuanto mayor es la exposición del espectador al espectáculo televisivo, tanto mayor es, en general, la influencia ejercida por el medio (Condry, 2002, p. 71).

Para otros, como Ferrés ${ }^{4}$ (1994), la televisión viene a sustituir la función materna y llega incluso a ocupar el lugar central en el diseño del hogar, y se convierte en punto de referencia obligado en la organización de la vida familiar. ${ }^{5}$ Además, -afirma-, sus efectos son inconscientes, inadvertidos, producidos sobre las emociones, y no sobre la razón de los individuos, por medio de estímulos que no son percibidos de manera consciente, ya sea por haber sido camuflados por el emisor, por existir desconocimiento de los códigos o una gran excitación emotiva en el receptor, por saturación de informaciones o porque las comunicaciones son indirectas (Ferrés, 1996, p.16).

Críticas provenientes del enfoque conductista y su visión del reflejo condicionado, según las cuales los medios de comunicación en general ejercen una influencia sobre una audiencia pasiva.

2 El potencial educativo de los medios de comunicación empezó a valorarse en los años 60 y 70 del siglo XX, con la radio y la televisión educativas utilizadas con fines escolares. El término paralelo se agrega luego al vislumbrar el potencial como medios de aprendizaje que tenían, no siempre en línea con los propósitos y contenidos del sistema de educación formal.

3 Pionero de la ciencia política y de las teorías de la comunicación, cuya visión fue superada luego por otras corrientes de pensamiento; pero que hoy, con el advenimiento y crecimiento de la Internet y las redes sociales, retoma protagonismo.

4 Español, doctor en ciencias de la información, especialista en comunicación audiovisual y educación; ha centrado sus trabajos en los temas de la socialización mediante comunicaciones inadvertidas y el de la educación en una cultura del espectáculo.

5 No resulta difícil pensar en "negociaciones" familiares para definir el programa por verse - si no se ha llegado a la opción de un televisor para cada uno-, así como en la ausencia de conversaciones familiares mientras está encendido; o en los programas que se ven como único tema de conversación. 
Según Maigret (2005, p. 93), por el contrario, "no se ha podido demostrar ninguna causalidad entre series de contenidos y de actitudes, y aun si en algunas investigaciones se pudieron obtener correlaciones (muchas veces contradictorias), estas no dicen nada de los factores complejos implicados allí, tales como el medio familiar y los valores sociales". Para Maigret, "el paradigma de los efectos fuertes es un paradigma débil porque aporta una información muy limitada sobre la realidad de la interacción social".

Otra de las grandes corrientes para analizar el efecto de los medios de comunicación sobre las personas es la denominada teoría crítica, desarrollada e impulsada por la Escuela de Frankfurt, ${ }^{6}$ que introduce el gran aporte de una reflexión sobre la ideología en el campo de la investigación sobre los medios, cambiando el reflejo condicionado por el efecto ideológico (Maigret, 2005).

La teoría crítica tiene entre sus tesis principales la existencia de una cultura de masas basada en el "bombardeo permanente de entretenimientos que afectan el juicio y adormecen la razón" a través de una industria de los medios de comunicación, que seducen porque "alivian, relajan, permiten soñar y tener esperanza", que transmiten estereotipos que simplifican el mundo y gustan por su "monotonía tranquilizadora". "Los medios forman una cortina de humo, un vapor que embrutece: la comunicación de masa conduce al silencio de la masa" (Maigret, 2005, pp. 110-111).

Para Eagleton (1991, en Stevenson, 1995, p. 144), más importante políticamente hablando que el efecto ideológico de la televisión durante el tiempo que se le mira, es el hecho de que, durante el tiempo que mira televisión "la gente no se compromete en una actividad política más seria", y que la televisión es "más una forma de control social que un aparato ideológico".?
El enfoque empírico para estudiar el efecto de los medios de comunicación en las audiencias aparece con Lazarsfeld, ${ }^{8}$ quien supera los métodos de análisis puramente reflexivos especulativos, incorporando la recolección de información y el análisis del comportamiento del público en estudios que dieron origen al término Mass Communication Research, 9 que lo llevaron a confirmar el peso de las relaciones interpersonales por encima de los medios en decisiones sobre bienes de consumo, la moda, el cine y asuntos públicos. ${ }^{10}$ Para él, "los efectos de los medios son indirectos y limitados, filtrados por las capacidades cognitivas de los individuos, difundidos horizontalmente al interior de las redes y no verticalmente del emisor hacia el receptor" (Maigret, 2005, p. 131).

El hasta aquí ignorado papel activo del receptor aparece con Hall (citado por Maigret, 2005), con quien el estudio deja de ser sobre los medios en sí mismos, o sobre su influencia como una variable de una sola dimensión, para pasar a ser sobre la recepción de los medios por parte de los públicos, sobre las interacciones sociales involucradas y sobre el contexto en que se desarrollan.

En el ámbito latinoamericano, Orozco (1996) plantea que es necesario precisar algunas situaciones relacionadas con las interacciones de la teleaudiencia, en un proceso de mediación múltiple. En ese contexto, se refiere por ejemplo a la "duración"11 del proceso de ver televisión, que él sostiene va más allá del tiempo transcurrido desde que una persona enciende el televisor hasta que lo apaga o se aleja de él.

El papel activo de las audiencias y el principio de la mediación múltiple está presente también en la obra de Fuenzalida (citado por Lozano, 2007, p. 192), quien realizó estudios que demostraban la importancia de la influencia grupal en el significado que las personas dan a los mensajes, destacando la influencia que la familia y los amigos ejercen

\footnotetext{
6 Integrada por filósofos judíos alemanes, en su mayoría obligados a emigrar a Ginebra en 1933, y luego a Nueva York en 1934, debido a las persecuciones de los nazis. Sus figuras preponderantes son Theodor Adorno y Max Horkheimer.

Comillas de Stevenson.

8 Paul Félix Lazarsfeld, sociólogo y psicólogo austríaco quien, al igual que los representantes de la Escuela de Frankfurt, se exilió en Estados Unidos después que aquellos, en 1935

Investigación sobre medios de comunicación masiva.

10 Trabajo que realiza con el apoyo de Eliu Katz, uno de sus estudiantes.

11 Comillas de Orozco.
} 
en los hábitos y preferencias televisivas de los jóvenes, así como la de las organizaciones de todo tipo, como fuentes de información y de elaboración de significaciones socioculturales.

En suma, el estudio de los medios está marcado por diferentes enfoques, contrapuestos entre sí algunos de ellos. Lo que no está en discusión es el hecho de que los medios de comunicación masiva, y, en el caso que atañe a este estudio, particularmente la televisión, son parte integral de la vida del ser humano de estos tiempos y, por tanto, su estudio $-\mathrm{y}$ el de su interacción con la audiencia- es relevante; pero con un sentido crítico desideologizado, que supere ideas preconcebidas $\mathrm{y} / \mathrm{o}$ convenientes a intereses propios.

Ineludiblemente, la existencia de un "problema" alrededor del consumo de la televisión en la vida "cotidiana"12 lleva a la cuestión del qué hacer. Y es aquí donde surge la opción de la llamada educación en medios, educación para la recepción, pedagogía crítica de los medios o educación para la televidencia, si se quiere ser más específico en cuanto al medio televisivo; términos que, esencialmente, se refieren al hecho de proporcionar a los individuos las herramientas para lograr un consumo crítico de los contenidos que ofrecen los medios de comunicación.

Al igual que el estudio de los medios, la educación, para su consumo, ha generado variados estudios y propuestas, como la de Fecé (2000), para quien los medios no actúan como espejos de la realidad, sino que producen construcciones de ella, por lo que "la enseñanza de los medios audiovisuales no se puede entender sin el complemento de otras acciones: las audiencias, estructura y funcionamiento de las industrias de comunicación, las prácticas periodísticas y el uso estético o creativo de los medios" (Tyner, 1996; citado en Fecé, 2000, p. 141).

Si de lo que se está hablando es de un acto educativo, sin duda es la escuela, en todos sus niveles, la llamada a construir nuevas relaciones con los medios de comunicación, ya que, como bien señala Buckingham (2005), los medios representan un "nuevo" ambiente de aprendizaje, un tipo de escolaridad informal, que para los jóvenes resulta mucho más atractiva que la obligatoria y aburrida aula tradicional. Lo que se propone es un uso pedagógico creativo y crítico de los medios y las nuevas tecnologías, que dé la posibilidad de "aprender de los medios" en lugar de "aprender por los medios", lo que permitiría apropiarse críticamente de sus contenidos y descifrar los cambios que conducen (González, 2000, p. 188).

\section{Diseño metodológico}

El estudio realizado es de tipo correlacional exploratorio, y constituye una aproximación al tema de la relación entre la televisión y las pautas de conducta entre los jóvenes salvadoreños, desde la perspectiva de los jóvenes mismos. La investigación se realizó con un enfoque cuantitativo, sin profundizar en las motivaciones detrás de dicha relación.

La técnica de investigación utilizada fue la encuesta, para la que se diseñó un cuestionario estructurado de diseño propio, que incluía 21 preguntas cerradas de opción múltiple, orientadas a obtener datos acerca de hábitos y preferencias de consumo del medio televisivo, así como de las conductas asociadas.

El instrumento de investigación fue validado con un investigador social y por medio de una prueba piloto, además de hacerlo con los encuestadores, previo a la capacitación que recibieron para su aplicación. El trabajo de campo estuvo a cargo de un equipo de 51 encuestadores, todos ellos estudiantes de comunicaciones de la Universidad Tecnológica de El Salvador, que fueron a su vez supervisados por el equipo investigador y docentes de la misma área, para cubrir todo el territorio nacional, utilizando los mismos criterios con los que trabaja el Centro de Investigación de la Opinión Pública Salvadoreña, CIOPS ${ }^{13}$

12 Comillas del equipo investigador. El término adquiere dimensiones sumamente complejas en tanto se refiere a un hecho materialmente invisible, pero presente; un hecho marcado - según Silverstone- por tensiones entre angustia y seguridad, entre actividad y pasividad, entre creatividad y adicción, entre lo público y lo privado, entre la dependencia y la independencia, y entre consumo y producción.

13 El CIOPS es el centro de investigación de la Universidad Tecnológica de El Salvador, que cuenta con más de veinte años de experiencia en la realización de sondeos de opinión pública a escala nacional. 
Como participantes del estudio se seleccionaron 1.207 jóvenes de ambos sexos, con edades comprendidas entre 15 y 24 años (según la definición aceptada por Naciones Unidas), localizados en las áreas urbanas y rurales del país, de los cuales, y en correspondencia con los números oficiales del último censo de población, ${ }^{14} 53,6 \%$ pertenece al sexo femenino y el $46,4 \%$ al masculino, mientras que el $78,6 \%$ habitan en el área urbana. En cuanto a la edad, el $55,3 \%$ están en el grupo etario entre 15 y 19 años, y el $44,7 \%$ restante entre 20 y 24 .

Para el cálculo de la muestra se utilizó la fórmula para poblaciones finitas. El tipo de muestreo utilizado es aleatorio no probabilístico para este subgrupo de la población. Los 1.207 jóvenes fueron ubicados y entrevistados en lugares públicos en 47 municipios de los catorce departamentos del país, representativos por su cantidad de habitantes.

Para el procesamiento de los datos se utilizó el programa SPSS, a través del cual se obtuvieron las frecuencias respectivas, así como las correlaciones de interés del equipo investigador.

\section{Resultados}

Evidentemente, la televisión domina las preferencias mediáticas, ya que aparece como el primer y el segundo medio preferido por los jóvenes, aunque sin ejercer un dominio absoluto, ya que su preferencia se presenta un poco por debajo de la mitad, mientras que la Internet destaca como la primera opción para una tercera parte de los jóvenes sujetos de estudio, y como segunda preferencia para dos de cada diez. La radio se ubica en el tercer lugar de las preferencias, para que los medios que implican el hábito de la lectura (prensa escrita y revistas) aparezcan como las últimas preferencias, salvo en el caso del tercer medio preferido, cuando la prensa escrita se posiciona en segundo lugar, por detrás de la radio (tabla 1 ).

TABLA 1.

Medio preferido, según orden de importancia

\begin{tabular}{lccc}
\hline \multicolumn{1}{r}{ Opciones } & \% Primera preferencia & \% Segunda preferencia & \% Tercera preferencia \\
\hline Prensa escrita & 7,4 & 11,7 & 25,2 \\
Radio & 15,3 & 28,6 & 30,0 \\
Televisión & 44,5 & 37,3 & 14,0 \\
Revistas & 0,9 & 3,0 & 9,4 \\
Internet & 31,8 & 19,4 & 21,4 \\
\hline
\end{tabular}

Sobre los motivos para encender el televisor, la búsqueda de entretenimiento es la razón que prevalece, con el $65,6 \%$ de las menciones. No llegan a una cuarta parte quienes afirman que esperan encontrar información, y apenas uno de cada diez busca educación. Estos resultados son preocupantes, ya que se está frente a una población joven que tampoco lee -el medio informativo y analítico por excelencia-: recuérdese que la prensa escrita no destaca ni en la primera ni en la segunda preferencia mediática, y no es sino hasta en la tercera preferencia que puntúa en segundo lugar.

\footnotetext{
14 Proyecciones de la Dirección General de Estadística y Censos, Digestyc.
} 
En cuanto a los hábitos de consumo de la televisión, la sala es el lugar mencionado por siete de cada diez jóvenes como el lugar de la casa en el que suelen ver la televisión, seguido del dormitorio, con el $26,4 \%$ de las menciones. El dato anterior sería positivo, si se rescata el supuesto de que la sala es el lugar de la casa que propicia el encuentro familiar. No obstante, no siempre la televisión la ven acompañados por alguien, y la tendencia a ver televisión en soledad es significativa.

Los padres, quienes se supone son los llamados - por edad, experiencia y responsabilidad natural y social- a guiar el proceso formativo de sus hijos, no son la compañía preferida por los jóvenes para ver la televisión, lo que podría explicarse por la diferencia de gustos que la brecha generacional impone. Sumado a lo anterior, cerca de seis de cada diez jóvenes reconocen que es poco lo que conversan con quienes los acompañan mientras ven televisión. Pero conversar no implica en sí mismo el acto de analizar con esas mismas personas los contenidos de los programas que se ven, y no llega a la mitad $(46,9 \%)$ el número de quienes dicen hacerlo de vez en cuando, y solamente una cuarta parte $(27,0 \%)$ afirma hacerlo casi siempre.

Motivo de atención son las cifras de más de siete de cada diez jóvenes a quienes la afición - ¿o adicción?_- por algunos programas de televisión los lleva, en mayor o menor medida, a dejar de realizar otras actividades, tales como labores del hogar, estar con amigos y estudiar (ver tabla 2).

TABLA 2.

Interferencia de la televisión con otras actividades

Opciones

Porcentaje

Frecuencia con que dejan de hacer otras actividades para no perderse programas

favoritos de televisión.

Siempre

Casi siempre

De vez en cuando

Nunca

Tipo de actividades que dejan de hacer para no perderse programas

favoritos de televisión.

Estudiar

Labores del hogar

Familiares

Estar con amigos

Otros 
Sobre la relación que pueda existir entre el consumo de televisión por parte de los jóvenes y sus pautas de conducta, merecen verse en detalle los resultados que se refieren a la incorporación de palabras al hablar cotidiano y a los cambios de opinión sobre temas específicos como efecto de la televisión (Ver tabla 3).

TABLA 3.

Incidencia de la televisión en pautas de conducta y opiniones

\begin{tabular}{lc}
\hline \multicolumn{1}{c}{ Opciones } & Porcentaje \\
\hline Repetición de palabras escuchadas a personajes de la televisión. & 50,3 \\
Sí & 47,0 \\
No & 2,0 \\
Sin opinión & \\
& \\
Cambio de opinión sobre algo luego de ver un programa de televisión. & 57,2 \\
Sí & 40,3 \\
No & 2,2 \\
Sin opinión & \\
\hline
\end{tabular}

Los datos de la tabla 3 permiten afirmar que es innegable que existe una relación entre el consumo de televisión por parte de los jóvenes y la manera en que estos hablan y piensan. Si se trata de una influencia directa, los mismos jóvenes valoran esa influencia como positiva, calificativo que la mitad de los entrevistados dio a la influencia de la televisión en su vida diaria.

De lo que se habla en el párrafo anterior es en definitiva de procesos de aprendizaje, y recuérdese que: “El aprendizaje no requiere de intencionalidad. El aprendizaje puede ocurrir sin el objetivo de aprender... Aprender no se restringe al uso de elementos racionales. Gran cantidad de lo que los educandos aprenden es a través de las emociones" (Charles, 1990, p. 71).

Si se analizan los datos a la luz de la estadística, es posible establecer correlaciones positivas significativas entre sí, aunque entre débiles y moderadas ${ }^{15}$ solamente entre aquellos aspectos que tienen que ver con la aceptación de incorporar a su vida diaria expresiones, conductas, apariencia y cambiar de opinión, en relación con el consumo de televisión. ${ }^{16}$

En ese sentido, el estudio no encontró asociación entre variables sociodemográficas como la zona de residencia, el sexo, la edad, condición social, religión, ocupación y nivel educativo, y los hallazgos en términos de hábitos de consumo de televisión, como son las preferencias por quiénes se supone son los llamados - por edad, experiencia y responsabilidad natural y social- a guiar el proceso formativo de sus hijos canales y programas, lugar y condiciones en que se ve televisión, así como la discusión que pueda generarse alrededor de sus contenidos.

Tampoco hay asociaciones entre esas mismas variables y las motivaciones para el consumo del medio con el hecho de dejar de realizar otras actividades para no dejar de ver

\footnotetext{
5 El nivel de correlación es débil si la correlación es de 0.0 a 0.2, y de débil a moderada si es de 0.2 a 0.4 .

16 Preguntas de la No. 18 a la No. 22 del formulario utilizado.
} 
televisión, o con la repetición de palabras o expresiones, comportamientos, apariencia o, incluso, cambios de opinión producidos luego de ver televisión. La misma ausencia de relación se da con la valoración — positiva para la mitad de los jóvenes, como se señaló antes- que hacen de la influencia de la televisión en su vida diaria.

\section{Conclusiones}

Evidentemente, alguna relación existe entre el consumo de la televisión por parte de los jóvenes salvadoreños y sus pautas de conducta, relativas a incorporar en su vida diaria expresiones, conductas, apariencia y cambios de opinión sobre temas específicos, pero no se llegaró a establecer asociaciones entre ellas. Esta situación permite concluir que, tal como sostiene Maigret (2005), para analizar el fenómeno de la influencia de la televisión en la vida de los jóvenes hay que considerar las interacciones sociales involucradas y el contexto en que se desarrollan.

Los resultados apuntan a que la interacción entre la televisión y las audiencias no es unidimensional, y, por el contrario, es compleja; que, tal como sostiene Orozco (1996), esa mediación puede originarse en la cultura, en la política, en la economía, en la clase social, en el género, en la edad, en la etnicidad, en los medios de información, en las condiciones situacionales y contextuales, en las instituciones y en los movimientos sociales, así como en la mente del sujeto, en sus emociones y en sus experiencias.

Sobre las preferencias mediáticas, se confirmó el dominio de la televisión como fuente de entretenimiento; pero es un hecho el crecimiento de la Internet. Lo que sí debería ser motivo de preocupación es el bajo nivel de consumo de la prensa escrita por parte de los jóvenes, en tanto medio informativo y reflexivo por excelencia. Predomina el gusto por lo liviano y la menor exigencia de espíritu crítico en jóvenes que están destinados a buscar su sobrevivencia en un ambiente laboral en el que pudieran estar en desventaja, por su carencia de competencias vitales para la toma de decisiones.

Nótese también la hora en que los jóvenes acostumbran ver televisión -en la noche- y la relación que podría tener con un tema de salud, tanto física como mental, dada la posibilidad de estarle "robando horas al sueño". Recuérdense los problemas de salud mental que sufre el país y los criterios médicos que recomiendan ocho horas de sueño como una de las medidas para conservarla. Y qué decir de la costumbre arraigada de comer al tiempo que se ve televisión, lo que expertos en nutrición señalan como posible causa de trastornos alimenticios y de obesidad.

En otro contexto, se señala a la televisión como capaz de congregar a toda la familia, lo que se complementa con su ubicación en la sala del hogar, que se supone es el lugar de encuentro familiar. Al aparente distanciamiento entre padres e hijos, expresado en el compartir o no el consumo de la televisión, súmense los conflictos que pudieran generarse de las actividades que los jóvenes dejan de hacer —labores del hogar y estudiar- para no perderse sus programas favoritos de televisión, que fácilmente tensionarían las en algunos casos - frágiles relaciones familiares y de poder construidas al interior de los hogares.

Hay que aclarar, sin embargo, que no se está planteando aquí que la televisión en sí misma sea el origen de los probables conflictos familiares o de la ausencia de comunicación. Como bien señala Grande (1999, p.148), “el probable problema primario de la TV está no tanto en el comportamiento que produce, aunque hay algún peligro en eso, sino en el comportamiento que evita: las pláticas intrafamiliares, los juegos e incluso las riñas familiares, a través de los cuales tiene lugar el aprendizaje del niño y con los cuales va formando su carácter".

Independientemente de las múltiples variables ligadas a la relación existente entre el consumo de televisión y algunas pautas de conducta de los jóvenes salvadoreños, la dimensión del fenómeno es tal que cae por su peso la necesidad de impulsar estrategias que posibiliten que los jóvenes "aprendan de los medios" y no "por los medios" (González, 2000).

Hasta aquí, es evidente que los resultados ilustran, pero falta profundidad en el estudio de las mediaciones televisivas. Su carácter cuantitativo, y el depender de respuestas frías de parte de los sujetos de estudio, no permite profundizar en su origen y motivaciones. $Y$ ante esa deuda pendiente, se impone la necesidad de seguir profundizando en el análisis y 
explorar la posibilidad de continuar y ampliar este esfuerzo investigativo con un enfoque cualitativo.

En otro orden, los autores se pronuncian por la promoción de una cultura de consumo de medios - y de la televisión en particular- que permita una participación "más activa" de la audiencia, en términos de decisiones y análisis; se habla de una iniciativa para proporcionar a los jóvenes las herramientas para lograr un consumo crítico de los contenidos que ofrecen los medios de comunicación, que no está reñido con las posibilidades de entretenimiento, ni supone el consumo exclusivo de espacios considerados educativos. "Se trata de aprender sin negar el placer. O, si se prefiere, de aprender desde el placer y gracias al placer" (Ferrés, 1994).

Si se trata de un esfuerzo sistemático de tipo educativo, la organización llamada a trabajarlo es la escuela, entendida esta como la instancia formal que, en todos los niveles (desde la educación inicial hasta la superior) y sectores (público y privado), propicia el aprendizaje para la vida personal y profesional de los individuos. Sin embargo, no se oculta tampoco el hecho de que, hoy por hoy, la escuela salvadoreña, desde sus máximas instancias rectoras hasta los llamados naturalmente a implantarla —los docentes-, no está preparada para asumir este reto.

Y es que no debe olvidarse que la adquisición de sentido crítico — cualidad que se aprende en la práctica - es inherente a un proyecto de educación en medios que, si bien es cierto se centra en el alumno, implica para el docente la obligación de ser crítico consigo mismo, de las prácticas comunicativas que utiliza, de los métodos y recursos didácticos que emplea (Charles, 1990). De ahí que, el sentido crítico frente a los medios debe ser adquirido primero por el docente.
Lo colosal de la tarea descarta esfuerzos institucionales aislados. Lo que se requiere es una política y un plan de nación que se cuide de caer en los riesgos de la regulación o, peor aún, de la censura. Se habla entonces de un esfuerzo estatal, por la cobertura y alcance que le son propios como por el poder institucional que detenta; que sume a todos los actores involucrados, los medios mismos incluidos.

Punto álgido de la propuesta es el cómo educar para los medios, por cuanto este es un aspecto que no puede dejarse a la casualidad, y requiere - por lo menos- la incorporación del tema al currículo, la definición de un método y la formación del docente. Para pensar en una propuesta de educación en medios hay que virar la vista hacia las experiencias previas en ese sentido. Europa y Suramérica son ejemplos que se deben analizar, ya que, más allá de las obvias diferencias culturales, el camino recorrido es guía para aquel que se inicia en esta ruta.

Por lo planteado hasta aquí, se sobreentiende que este programa de educación en medios no puede ser producto de la casualidad, de la improvisación o del solo entusiasmo de alguno de sus actores. Si como sociedad se ha satanizado y acusado a los medios, especialmente a la televisión, de ser el origen de algunos de sus problemas, esa misma sociedad debe asumir con responsabilidad y seriedad la manera de relacionarse con ellos, no de enfrentarlos. Es el momento de trascender la simple crítica y la censura.

Desde las universidades, como espacios generadores de conocimiento, y con responsabilidades con su entorno, pudieran generarse propuestas concretas en este sentido $y$, atendiendo a su cometido de proyección social, relacionarse activamente con sus diferentes contextos y trabajar con una calidad vinculada con la pertinencia y responsabilidad con el desarrollo sostenible de la sociedad, impulsando un modelo académico que se caracterice por la indagación de los problemas en distintos contextos. ${ }^{17}$

17 Con base en el concepto de proyección social asumido por la Universidad Tecnológica de El Salvador. 


\section{Referencias}

Buckingham, D. (2005). Educación en medios. Alfabetización, aprendizaje y cultura contemporánea. Barcelona, España: Paidós.

Condry, J. (1998). Ladrona del tiempo, criada infiel. En Popper, K. y Condry, J. (Ed.). La televisión es mala maestra (pp. 67-95). México D.F., México: Fondo de Cultura Económica.

Charles, M. (1990). Educación para la recepción. Hacia una lectura crítica de los medios (Ed.), Aparato escolar y medios de comunicación (pp. 68-81). México: Editorial Trillas.

Ferrés, J. (1994). Televisión y educación. Barcelona, España: Paidós Ibérica, S.A. Buenos Aires, Argentina: Editorial Paidós, SAICF.

Ferrés, J. (1996). Televisión subliminal. Socialización mediante comunicaciones inadvertidas. Barcelona, España: Paidós Ibérica, S.A. Buenos Aires, Argentina: Editorial Paidós, SAICF.

Lozano, J. (2007). Teoría e investigación de la comunicación de masas. México, D.F., México: Pearson Educación.
Fecé, J. (2000). Comunicación y educación en la sociedad de la información. Nuevos lenguajes y conciencia crítica (Ed.), Lectura crítica de medios audiovisuales (pp. 135-168). Barcelona, España: Editorial Paidós.

González, J. (2000). Comunicación y educación en la sociedad de la información. Nuevos lenguajes y conciencia crítica (Ed.), Variables de la educación en comunicación (pp. 171-219). Barcelona, España: Editorial Paidós.

Grande, J. (1999). La influencia de la televisión. San Salvador, El Salvador: Editorial e Imprenta Universitaria.

Maigret, É. (2005). Sociología de la comunicación y de los medios. Bogotá, Colombia: Fondo de Cultura Económica.

Orozco, G. (1996). Televisión y audiencias. Un enfoque cualitativo. México, D.F.; Universidad

Stevenson, N. (1995). Culturas mediáticas. Teoría social y comunicación masiva. Buenos Aires, Argentina: Amorrortu Editores S.A. 